\title{
: Hallazgos Preliminares del Ensayo Clínico Multicéntrico de Evaluación de la Seguridad de la Aspiración Supraglótica Continua mediante el Nuevo Dispositivo de Invención SUPRAtube para Paciente Intubados y Ventilados Mecánicamente
}

Mauricio Orozco-Levi, Melissa Mogollón, Javier Fajardo, Alba Ramírez-Sarmiento, Rafael Conde, Héctor Ortega, Manuel Pacheco, Luis Echeverría, Jaime Rodríguez, Federico Saaibi, Alejandro Londoño. Servicio de Neumología, Centro para el Cuidado de la Salud Respiratoria, Hospital Internacional de Colombia. Santander, Colombia. Universidad de Santander, Colombia. Universidad Industrial de Santander (UIS), Bucaramanga, Colombia. IPS Respiremos, Pereira, Colombia. Clínica CardioVid, Medellín, Colombia. Fundación Neumológica Colombiana, Bogotá, Colombia.

\section{INTRODUCCIÓN Y OBJETIVO}

La Hipertensión Arterial Pulmonar (HAP) es un trastorno hemodinámico multifactorial crónico que impone un grave impacto tanto al paciente como su entorno, así como al sistema de salud estatal. En Colombia, la terapia específica con medicamentos (orales, inhalados o parenterales) ofrece 14 diferentes de ellos, y la posibilidad de 84 combinaciones que actúan sobre tres vías metabólicas definidas (i.e., endotelina, óxido nítrico y prostaciclina). Existe en la actualidad una iniciativa gremial multidisciplinaria y multicéntrica que consolida la información de pacientes con diagnóstico confirmado hemodinámicamente de HP, con especial énfasis en los Grupos 1 y 4 , llamada Red Colombiana de Hipertensión Pulmonar (HAPred.co), lo cual ha permitido el análisis de la respuesta ante los tratamientos específicos por los pacientes en nuestro país.

Objetivo: Evaluar el tipo y magnitud de cambio(s) hemodinámicos y funcionales relacionados con el tratamiento crónico con fármacos específicos para HAP en pacientes con diagnóstico incidente de la enfermedad (grupos 1 y 4)en la zona andina colombiana.

\section{MATERIALES Y MÉTODOS}

Se analizaron como variables independientes el inicio del tratamiento y su duración, y mientras que las presiones de arteria pulmonar (PAP sistólica, diastólica y media) se incluyeron como como variables dependientes. Se analizaron modelos de correlación lineal y no-lineal respecto del cambio tanto en términos de valores absolutos como en porcentaje de cambio (post-pre/pre, \%) entre el estudio hemodinámico invasivo (diagnósticoreferente) y el último cateterismo disponible en el seguimiento (final).

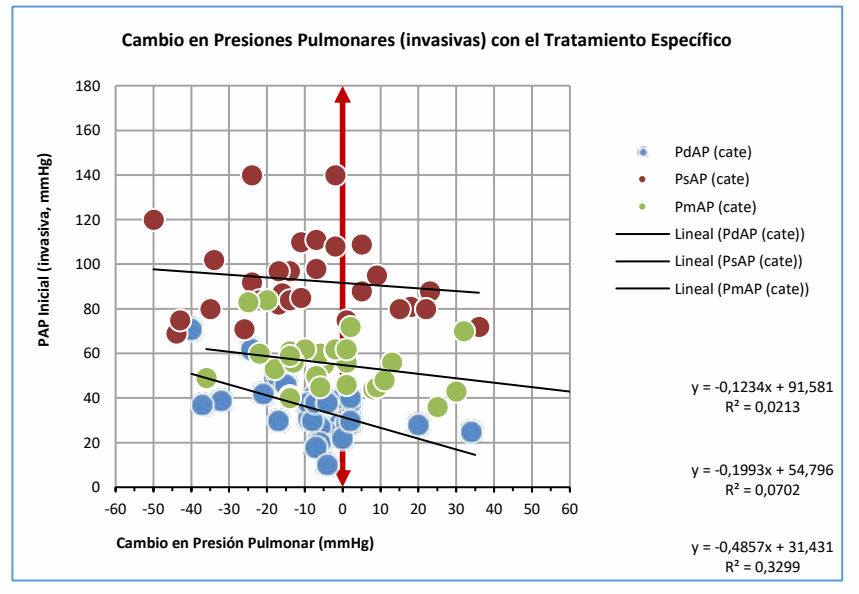

Figura 1: Gráfico de dispersión entre las variables de Presión Pulmonar al Inicio vs. Cambio en las mismas ante el Tratamiento Especifico. Las líneas continuas representan la correlación lineal en cada caso.

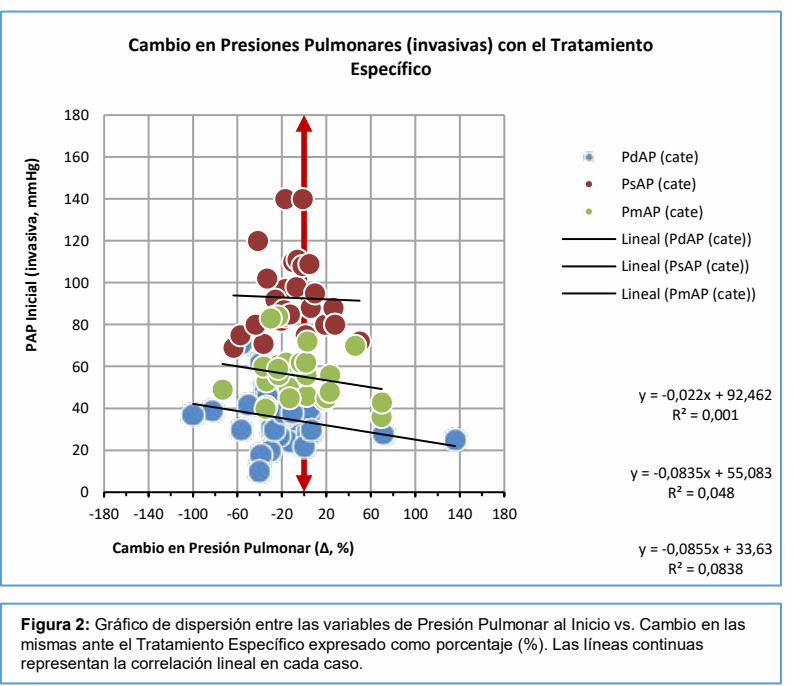

\section{RESULTADOS}

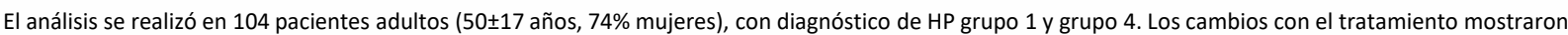
un amplio rango, con PAPs $=-9 \pm 26 \%$, PAPd $=-18 \pm 42 \%$ y PAPm $=-5 \pm 31 \%$. Esta dispersión se justificó por la existencia de tres grupos de pacientes: unos sin cambio en las presiones pulmonares (i.e, no-respondedores), otros con empeoramiento de estas (también no-respondedores), y otros (50\% del total) que por el contrario mostraron mejoría (disminución de $10 \%$ o más del valor inicial) de la presión pulmonar media (figuras 1, 2 y 3 ). No se evidenció una función lineal de correlación con el tiempo de tratamiento $\left(p=n s, r^{2} \leq 0,1\right)$. El ajuste por edad no mostró mejoría de la correlación.

\section{CONCLCUSIONES}

En pacientes con HAP en la región andina colombiana, la respuesta ante los fármacos específicos aprobados no es homogénea en magnitud ni sentido en todos los pacientes. Se identifican pacientes respondedores (aproximadamente $50 \%$ ) y otros no-respondedores en términos de presión de arteria pulmonar, independientemente del tiempo de tratamiento. Estas evidencias resaltan la necesidad de evaluar de forma objetiva (invasiva) la hemodinámica pulmonar para identificar los pacientes que requerirán estrategias de escalonamiento o combinaciones de tratamiento, incluyendo el trasplante pulmonar. 